\title{
Results for Initial Trials comparing Conformal with Non-Conformal Hypocenter Scanning Algorithms
}

\author{
George R. Daglish
}

\section{ABSTRACT}

This present paper sets out to perform a limited comparison between that method of P2P (point to point) scanning for Hypocenters, which uses the structure, imparted by a conformal mapping, to accelerate its ray tracing activity, and a method of scanning which incorporates the Earth spheroidal geometry directly within its ray tracing technique and which does not currently use a conformal mapping technique. The "conformal" method uses a mean-radius spherical Earth or can take a mean radius over the region covered by Epicenter and the sensor-field.

In this comparison an attempt is made to compare and contrast a set of some possible functions ("indicator functions") which serve to define the position of a Hypocenter within their response curve which is formed during the scan. Depending on the function, the expected indication may be either a minimum or a maximum, occurring within the range of the response.

Keywords: Hypocenter, Scan, Conformal mapping, Spheroidal Earth Geometry, Indicator Function.

\section{INTRODUCTION}

This paper resumes a set of trials for scanning methods to determine hypocenter depths (denoted by $H_{d}$ ) once the epicenter (denoted by $\bar{\epsilon}$ ) of the event is known. The epicenter may be known in several ways [1][2]. These trials are formed into three groups, each processing data from three known earthquakes:

1. Conformal Scan with Static Granularity

2. Conformal Scan with Dynamic Granularity

3. Spheroidal Scan with Geocentric vectors

For the record, the program structures used, for groups 1 and 2, were:

Solution: Qloc00

Program: Qloc00RayDuff06DSW.cpp

h.file: VWplane TransformWX.h

Algorithm: WplaneDeltaRGLN(...)

and, for group 3:

Solution: EpiHypo0!

Program: ETTST 16EW6.cpp

$\mathrm{h}$ file: QlocEllipse04Singlev.h

Algorithm: HypocenterSpheroidScan(...)

TABLE I: EARTHQUAKE PARAMETERS

\begin{tabular}{lllll}
\hline \hline Number & Region & $\begin{array}{l}\text { Magnitude } \\
{[\mathrm{M}(\mathrm{w})]}\end{array}$ & $\begin{array}{l}\text { Epicenter } \\
\text { Lat/long } \\
(\text { degrees })\end{array}$ & $\begin{array}{l}\mathrm{H}[\mathrm{d}] \\
(\mathrm{km})\end{array}$ \\
\hline 1 & West of North & 5.4 & $41.7136 \mathrm{~N} /$ & 9.9 \\
\hline \hline
\end{tabular}
from reference [3].
Published Online: March 6, 2020

DOI : $10.24018 / 2020.1 .2 .2$

George R. Daglish *

Independent Analysis and Computation Ltd., Camberley, Surrey, UK.

(email: george@iaac.co)

*Corresponding Author

\begin{tabular}{lllll}
\hline \hline & American Coast & \multicolumn{3}{l}{$126.844 \mathrm{~W}$} \\
\hline 2 & Fiji Islands Region & 4.5 & $\begin{array}{l}17.8997 \mathrm{~S} / \\
178.5269 \mathrm{~W}\end{array}$ & 558.9 \\
\hline 3 & Just East of Honshu & 5.4 & $\begin{array}{l}35.621 \mathrm{~N} / \\
140.6862 \mathrm{E}\end{array}$ & 36.0 \\
\hline 4 & South Mid-Atlantic & 5.2 & $\begin{array}{l}47.186 \mathrm{~S} / \\
13.430 \mathrm{~W}\end{array}$ & 10.0 \\
\hline 5 & Trench & 4.1 & $\begin{array}{l}3.9999 \mathrm{~N} / \\
123.3981 \mathrm{E}\end{array}$ & 495.1 \\
\hline 6 & Celebes Sea & 6.1 & $\begin{array}{l}27.446 \mathrm{~N} / \\
103.42 \mathrm{E}\end{array}$ & 10.0 \\
\hline 7 & Yunnan (China) & 4.7 & $\begin{array}{l}12.2398 \mathrm{~S} / \\
167.135 \mathrm{E}\end{array}$ & 259.3 \\
\hline \hline
\end{tabular}

The basis for the conformal scan is a conformal transform, $w=\ln (v)$, which is depicted in the following diagram (after Schaum "Complex Variables").

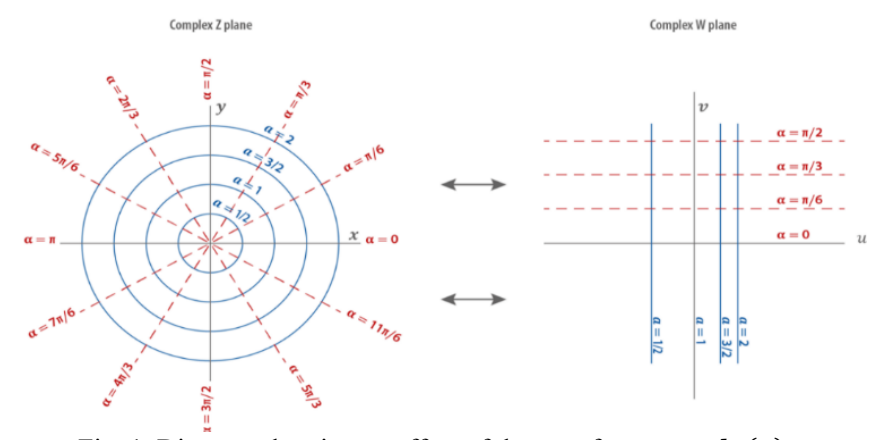

Fig. 1. Diagram showing an effect of the transform: $\boldsymbol{w}=\ln (\boldsymbol{v})$

The next four paragraphs, and the Fig. 1, above, are quoted 
There are four points to be made in the approach to the Conformal algorithm currently under consideration:

1. The P2P ray-trace algorithms used here are associated with flat, layered and parallel strata. There are two guises for this form: (a) Incremental; (b) Analytic. The analytic form is not considered here. If we take the simple view towards constructing the $\mathrm{P} 2 \mathrm{P}$ algorithm incrementally by radius or by subtended angle, we can generate fast algorithms using a form of Lagrange control, which will provide $\mathrm{P} 2 \mathrm{P}$ traces very rapidly. For $\mathrm{P} 2 \mathrm{P}$ tracing in parallel strata, the Lagrange control method can be deployed as will be described'

2. It is possible by the transform $w=\ln (v)$, to return a discoid in the $\mathrm{v}$-plane to a rectangular area in the w-plane (see Fig. 1).

3. Following on from the given illustration it may be seen that the orthogonal coordinate axes in the wplane can be returned to the z-plane as plane polar coordinates giving the structure for the discoid. On the w-plane we may proceed to perform P2P tracing as within a set of parallel superimposed strata, remembering that the $\mathrm{w}$-plane real axis is the logarithm of any radial length, while the imaginary axis is linear in angular measure.

4. On performing the inverse transform into the vplane onto the discoid region, centered on the origin, it is possible to extract, from this domain space, values for the applied velocity model and hence the travel-time of the trace as well as its pathlength. The take-off angle can also be formed from data within the w-plane and bundled with the v-plane information.

\section{CONFORMAL SCANNING}

Following on from point 3 , above, that the measure of the real axis in the w-plane, that acts as the rectilinear frame for the P2P activity, is logarithmic, we find that if the subsequent depth increments are calculated as:

$$
\delta \rho_{i}=\ln \left(L_{i-1}\right)-\ln \left(L_{i-1}-\delta \rho_{0}\right)
$$

or:

$$
\delta \rho_{i}=\ln \left(L_{i-1}+\delta \rho_{0}\right)-\ln \left(L_{i-1}\right)
$$

(where $\delta \rho_{0}$ is a basally chosen interval, and $L_{I}$ the real lengths corresponding to the radii in the v-plane) then the contraction of interval experienced in such a sequence may not allow proper arithmetic to take place. Therefore, appropriate Granularity must be calculated for each depth point to allow the series to run to completion without registering an exception condition.

The imaginary axis in the w-plane is linear in the angular displacement around the origin in the v-plane and each angular increment, $\delta \theta_{i}$, is calculated as:

$\delta \theta_{i}=\delta \rho_{i} \cdot \tan \left(\psi_{i}\right)$

on the upwards path, or as:

$$
=\delta \rho_{i} \cdot \tan \left(\pi-\psi_{i}\right)
$$

on the downward. Here, $\psi_{i}$ is the conformal take-off angle relative to the current parallel to the real axis (radius in $\mathrm{v}$ plane).

We accumulate as:

$$
l=\sum \delta \theta_{i}
$$

Here, let $\lambda$ be the intended target distance (i.e. angular distance between epicenter and given station), and $l$ the partial target value achieved by the ray for a given value of $\psi$. We write

$\left.\varepsilon_{\lambda}\right|_{i}=\frac{\lambda-l}{l}$

$\psi_{i+1}=\psi_{i} \cdot\left(1-\frac{\left.\varepsilon_{\lambda}\right|_{i}}{n_{i}}\right)$

where $\left.\varepsilon_{\lambda}\right|_{i}$ is the multiplying factor at the juncture between the $i^{\text {th }}$ and the $(i+1)^{t h}$ iteration. $n_{i}$ may be the number of steps the ray tracer portion of the algorithm has taken to

\begin{tabular}{|c|c|c|c|c|}
\hline $\begin{array}{l}\text { Indicato } \\
\text { r code }\end{array}$ & $\begin{array}{c}\text { Static } \\
\text { Granularit } \\
\mathbf{y}\end{array}$ & $\begin{array}{c}\text { Dynamic } \\
\text { Granularit } \\
\mathbf{y}\end{array}$ & $\begin{array}{c}\text { Non- } \\
\text { Conformal } \\
\text { [Spheroidal } \\
\text { \&Geocentric } \\
\text { ] } \\
\end{array}$ & $\begin{array}{c}\text { Earthquak } \\
\mathrm{e}\end{array}$ \\
\hline 01D & 480 & 540 & $?$ & E02 \\
\hline $01 \mathrm{~F}$ & $480 / 640$ & $510 / 650$ & 466 & \\
\hline 02D & 480 & 560 & 435 & $H_{d}$ \\
\hline 02F & 460 & 510 & 433 & $=558 \mathrm{~km}$ \\
\hline 03H & 430 & 480 & 416 & \\
\hline 03I & 470 & 500 & 458 & \\
\hline $04 \mathrm{~F}$ & 610 & 650 & 512 & \\
\hline 05G & 580 & 570 & 483 & \\
\hline $05 \mathrm{~N}$ & 530 & 550 & 438 & \\
\hline 01D & 410 & 420 & $?$ & E05 \\
\hline $01 \mathrm{~F}$ & $410 / 750$ & $430 / 780$ & 400 & \\
\hline 02D & 410 & 420 & 400 & $H_{d}$ \\
\hline $02 F$ & 410 & 420 & 400 & $=495 \mathrm{~km}$ \\
\hline 03H & 400 & 420 & 416 & \\
\hline $03 I$ & 410 & 420 & 466 & \\
\hline $04 \mathrm{~F}$ & 690 & 720 & 533 & \\
\hline 05G & 410 & 440 & $?$ & \\
\hline $05 \mathrm{~N}$ & 410 & 420 & 500 & \\
\hline 01D & 630 & 650 & 291 & E07 \\
\hline $01 F$ & $? / ?$ & $? / ?$ & 291 & \\
\hline 02D & 650 & 650 & 283 & $H_{d}$ \\
\hline 02F & $? / ?$ & $? / ?$ & 300 & $=259 \mathrm{~km}$ \\
\hline 03H & 600 & 610 & 283 & \\
\hline 03I & 620 & 650 & 275 & \\
\hline $04 \mathrm{~F}$ & 830 & 860 & 283 & \\
\hline 05G & $? / ?$ & $? / ?$ & 283 & \\
\hline $05 \mathrm{~N}$ & 710 & 730 & 450 & \\
\hline
\end{tabular}
achieve the off-target position $l$ at the $i^{\text {th }}$ iteration, or a given factor, or unity.

TABLE II: RESULTS 
[The "?" in this above table indicates "no distinguishable" minimum or maximum, and the "?/?" indicates the same where once there were two distinguishable minima.]

The Granularity, in these conformal scans for Hypocenter depth, $H_{d}$, can be arranged in two ways:

Static Granularity - Same number of intervals whatever the depth, this implies that the values of the scanning depth interval, $\delta \rho$, must be dynamic.

Dynamic Granularity - Varying number if intervals forming the changing depth during the scan, this implies that the values for the scanning interval, $\partial \rho$, must remain constant or static.

Static granularity parameters are generated by solving the equation below for $\delta \rho$ :

$$
\ln \left(\frac{L_{0}}{\delta \rho}\right)-\ln \left(\frac{L_{0}}{\delta \rho}-U n i t y\right)=\text { Zero }
$$

Dynamic granularity parameters are generated by solving the following equation for $\delta \rho$ :

$$
\ln \left(L_{0}\right)-\ln \left(L_{0}-\delta \rho\right)-\ln (\delta s)=Z e r o,
$$

Here $L_{0}$, (the depth at the given depth-point) and $\delta s$, (required magnitude for the ultimate scanning interval (defined as > Unity)) are known.

These equations are solved for each depth-point in the scan and supply the parameters for the P2P ray traces which each form the body of the scan, i.e. the set structure $-\left\{\boldsymbol{t}_{\boldsymbol{i}}\right\}_{j}$, that is made up from the "hypothetical" timings " $\mathrm{i}$ " originating from the depth-points “ $\boldsymbol{j}$ ".

The value discovered for parameter $\delta \rho$ is used as the basal depth increment, $\delta \rho_{0}$, for the scan from this depth point.

The P2P ray traces use a granularity defined by $\boldsymbol{i n t}\left(\left(\frac{L_{0}}{\delta \rho}\right)+\right.$ $\mathbf{0 . 5 )}$ in each case.

[Here: $\left\{\boldsymbol{\tau}_{\boldsymbol{i}}\right\}$ is the object timing set gathered at the active seismographs. This may be processed as "centered" or "basal". $\left\{\boldsymbol{t}_{\boldsymbol{i}}\right\}$ is the hypothetical timing set generated at a given depth-point and which also may be processed as "centered" or "basal".

\section{THE INDICATORS}

Each indicator is generated for each depth-point in the scan. These, in total, will form an "indicator response" (see figures from Fig. 5 onwards) in which an infimum or supremum may be taken to indicate the position of a Hypocenter focus, $H_{d}$, which then supplies an estimated depth co-ordinate for the event.

\begin{tabular}{|c|c|c|}
\hline Code & Name & Formula \\
\hline 01D: & 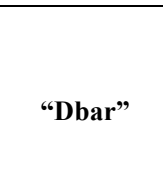 & $\begin{array}{r}\frac{1}{n} \cdot \sum\left|\left(\tau_{i}-t_{i}\right)\right| \\
d_{i}=\left|\tau_{i}-t_{i}\right| \\
D=\frac{1}{n} \cdot \sum d_{i}\end{array}$ \\
\hline 01F: & “SigmaD” & $\sqrt{\frac{1}{n}} \cdot \sum\left(D-d_{i}\right)^{2}$ \\
\hline 02D: & “ChiNS" & $\begin{array}{c}\frac{1}{S} \cdot(\text { Unity }+\sqrt{\text { Unity }-S * S}) \\
S=\frac{(S q-S u)}{S u} \\
S q=\sum\left(\tau_{i}+t_{i}\right)^{2} \\
S u=\sum\left(\tau_{i}^{2}+t_{i}^{2}\right)\end{array}$ \\
\hline 02F: & "ChiSM" & $S=\frac{(S q-S u)}{S u}$ \\
\hline 03H: & "ChiLnN" & $\ln \left(\frac{\chi^{2}}{n}\right):$ viz. $\ln \left(\frac{\sum \frac{\left(\tau_{i}-t_{i}\right)^{2}}{\tau_{i}}}{n}\right)$ \\
\hline 03I & "ChiNrM" & $\sum \tau_{i}^{2}-\sum t_{i}^{2}$ \\
\hline 04F: & “Fsh03” & $\frac{1}{2} \cdot\left(\ln S_{1}-\ln S_{2}\right) ; S_{1}>S_{2}$ \\
\hline 05G: & "LocalRms2 & $\frac{1}{n} \cdot \sum\left(D-\left(t_{i}-\tau_{i}\right)\right)^{2} ; \quad D=\frac{1}{n} \cdot \sum\left(t_{i}-\tau_{i}\right)$ \\
\hline
\end{tabular}

TABLE III: TABLE OF INDICATORS

\section{Alternative Methods}

A non-conformal method for forming $\mathrm{P} 2 \mathrm{P}$ ray-traces can consist of, say, the use of spherical earth geometry for a tabular scan using a mean earth radius, then subsequently, the natural spheroidal geometry of the Earth, as a constraint on a final least-squares calculation [4][5].

Using purely spheroidal geometry, a second approach for further non-conformal P2P scans is to drive the process from a set of points laid down on a geodesic line [which may also be rapidly calculated [6], on the given spheroid], each next point determining the subsequent position vectors that will be used to construct the next step in the trace. This is denoted as: $g_{i}, g_{i+1}$ in the following three diagrams. Given an appropriate take-off angle, then that P2P trace which coincides, within required accuracy limits, with the end point of the geodesic marker line of points [a 3-d space frame being used for point co-ordinates], is considered to be a hit and to represent the required $\mathrm{P} 2 \mathrm{P}$ trace.

The following three modes for this kind of P2P method: geocentric latitude position vector (GLPV); latitudinal radius position vector (LRPV) and normal latitudinal 
position vector (NLPV), all employing a spheroidal Earth, are depicted in Fig. 2, 3 and 4.

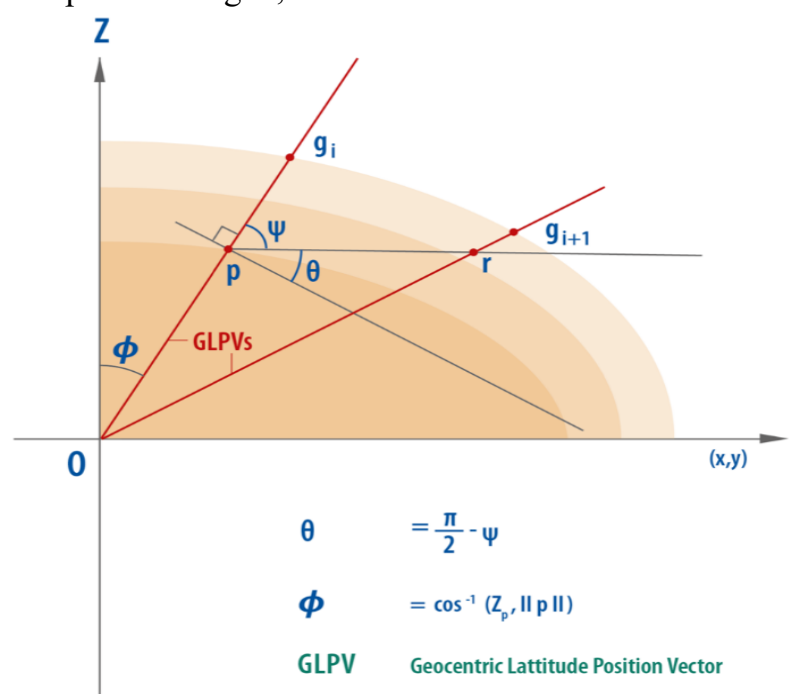

Fig. 2. GLPV ray trace, step construction

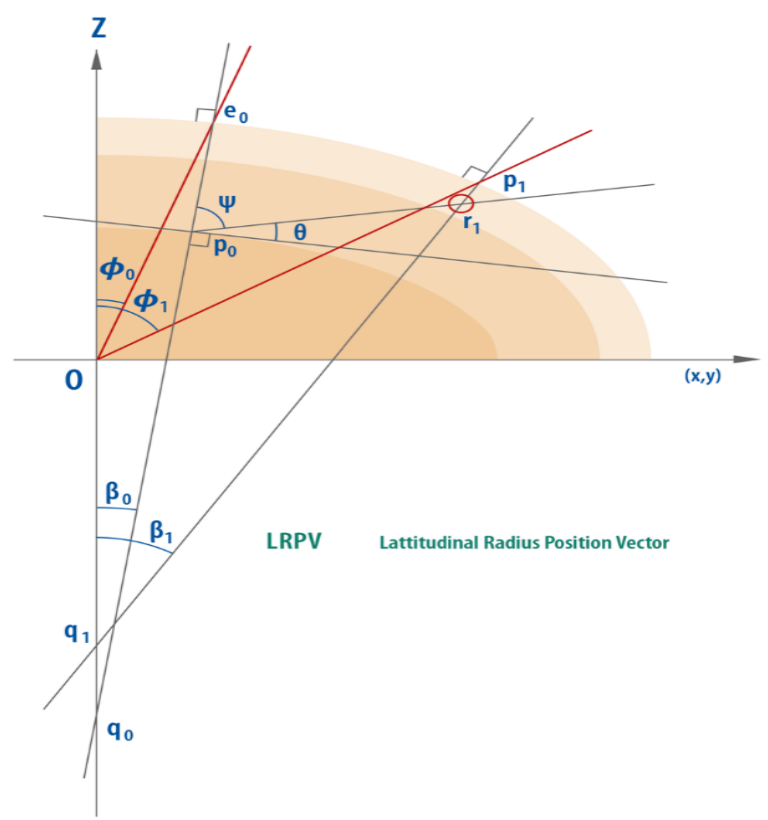

Fig. 3. LRPV ray trace, step construction

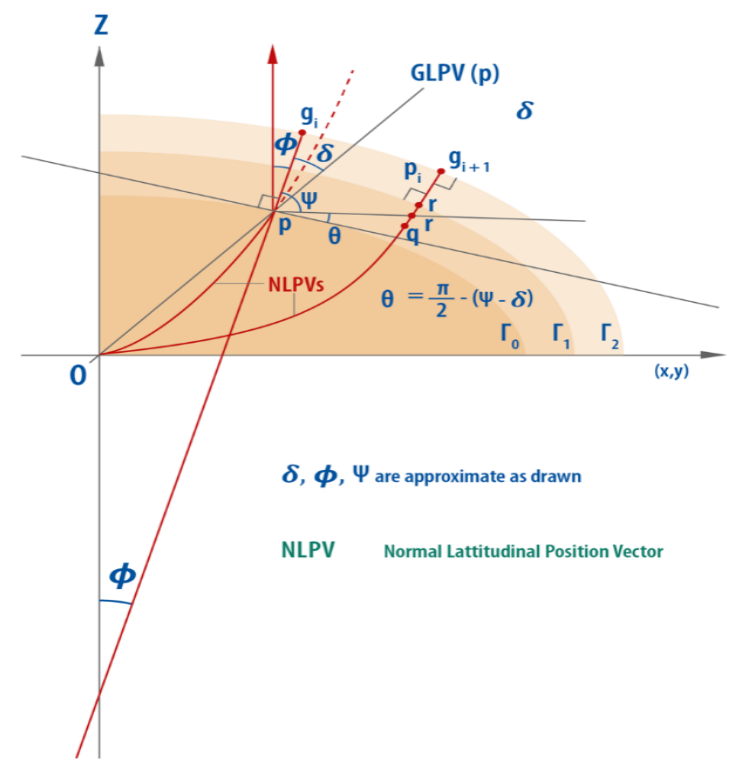

Fig. 4. NLPV ray trace, step construction
It is the GLPV method which has been used for this paper. One possible way to make the calculation which forms the step constructions for this mode is by an iterative Newton/Raphson scheme. We define this in the following.

Consider Fig. 2 and the vector $g_{i+1}$ with coordinates: $x_{g}, y_{g}$, $z_{g}$. The equation for the line defined by $g_{i+1}$ is:

$$
\frac{x-x_{g}}{0-x_{g}}=\frac{y-y_{g}}{0-y_{g}}=\frac{z-z_{g}}{0-z_{g}}=t .
$$

Thus:

$x=x_{g}(1-t) ; y=y_{g}(1-t) ; z=z_{g}(1-t)$.

Apply an intersection to the cone, formed from the point $p$ and the take-off angle $\psi$, having performed a yaw and a pitch, followed by a second yaw transform, on the given vector structure.:

$$
g_{i+1}^{\prime}=Y_{g} \cdot g_{i+1}
$$

where $Y_{g}$ is the final yaw matrix on the vector $g_{i+1}$,

$z_{g}^{\prime}(1-t)=\tan \left(\frac{\pi}{2}-\psi\right) \cdot \sqrt{(1-t)^{2} \cdot\left(x_{g}^{\prime 2}+{y_{g}^{\prime}}^{2}\right)}+\|p\|$

Letting the above function take the form $F(t)=$ Zero:

$$
z_{g}^{\prime}(1-t)-\tan \left(\frac{\pi}{2}-\psi\right) \cdot \sqrt{(1-t)^{2} \cdot\left(x_{g}^{\prime 2}+y_{g}^{\prime 2}\right)}-\|p\|=\text { Zero }
$$

We then have:

$$
\frac{\partial F(t)}{\partial t}=\frac{\left(x_{g}^{\prime 2}+{y_{g}^{\prime}}^{2}\right) \cdot(1-t) \cdot \tan \left(\frac{\pi}{2}-\psi\right)}{\sqrt{(1-t)^{2} \cdot\left(x_{g}^{\prime 2}+y_{g}^{\prime 2}\right)}}-z_{g}^{\prime}
$$

and we attempt to find the root of $F(t)$ by Newton/Raphson iteration as:

$$
t_{i+1}=t_{i}-\frac{F\left(t_{i}\right)}{\frac{\partial F\left(t_{i}\right)}{\partial t}}
$$

So now the intersection point denoted by " $\mathrm{t}$ " in the figure is given by:

$\left[\begin{array}{l}x_{g} \\ y_{g} \\ z_{g}\end{array}\right] \cdot(1-t)$

once it has been transformed by the inverse of the group of yaw and pitch matrices.

\section{Visualization of Results}

These results represent some responses of the Indicator coded as " $\mathbf{0 3 H}$ " in the above Table III. 


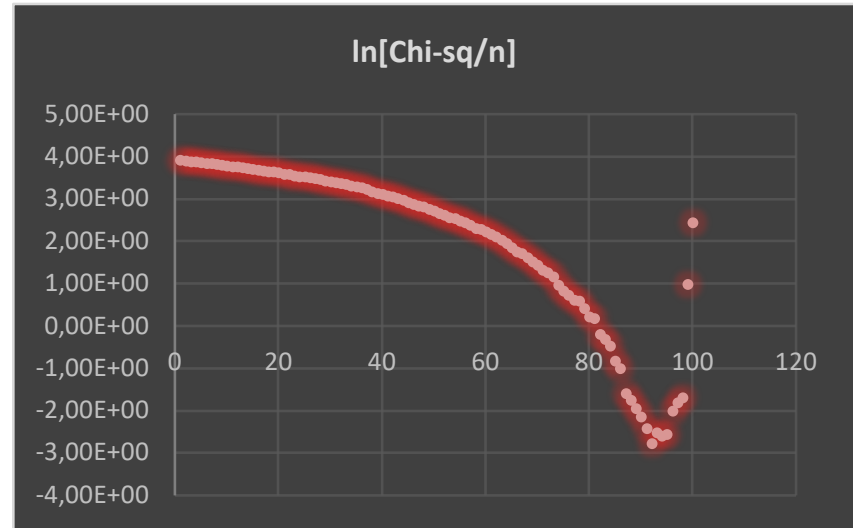

Fig. 5. E03 Spheroid. [Scan Depth $=1000$ km, on Spherical Earth.]

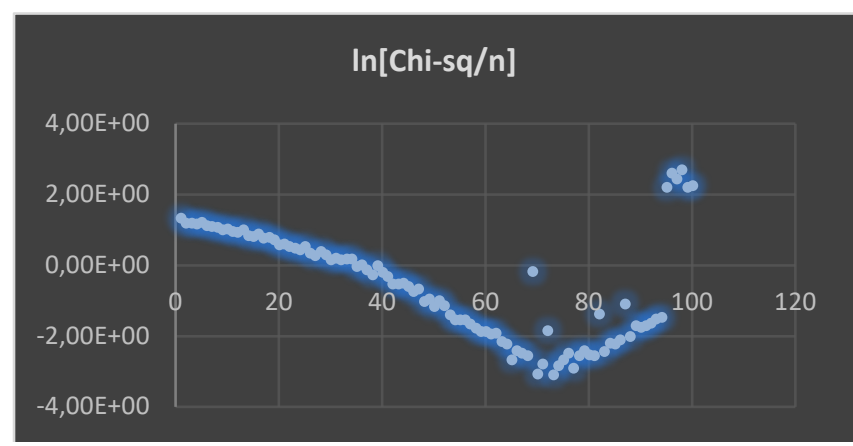

Fig. 6. E03 Spheroid. [Scan Depth $=300 \mathrm{~km}$, on Spherical Earth]

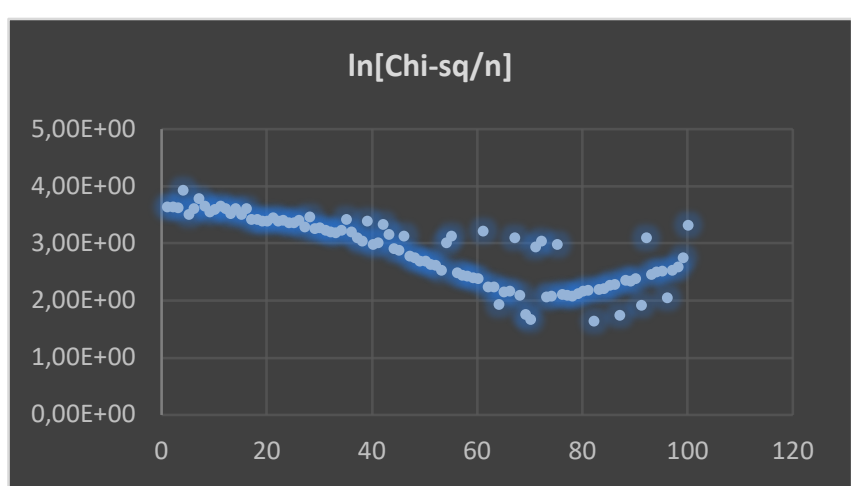

Fig. 7. E03 Spheroid. [Scan Depth $=1000$ km, on Spheroidal Earth]

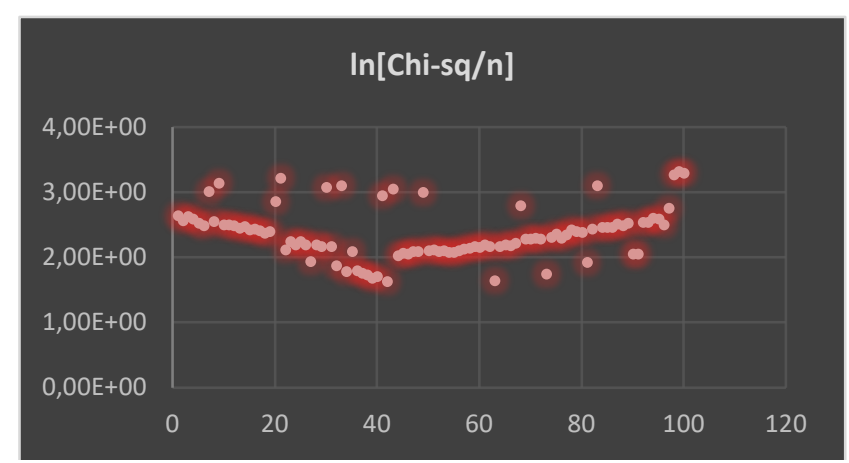

Fig. 8. E03 Spheroid [Scan Depth $=500 \mathrm{~km}$, on Spheroidal Earth]

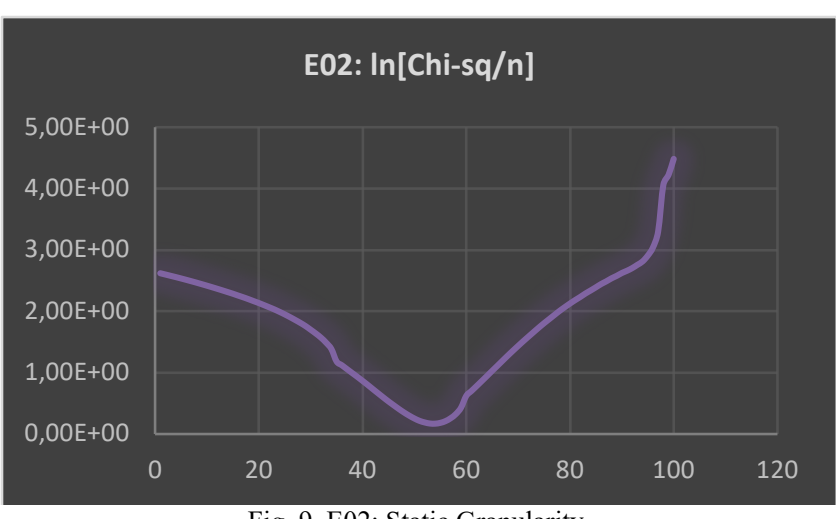

Fig. 9. E02: Static Granularity
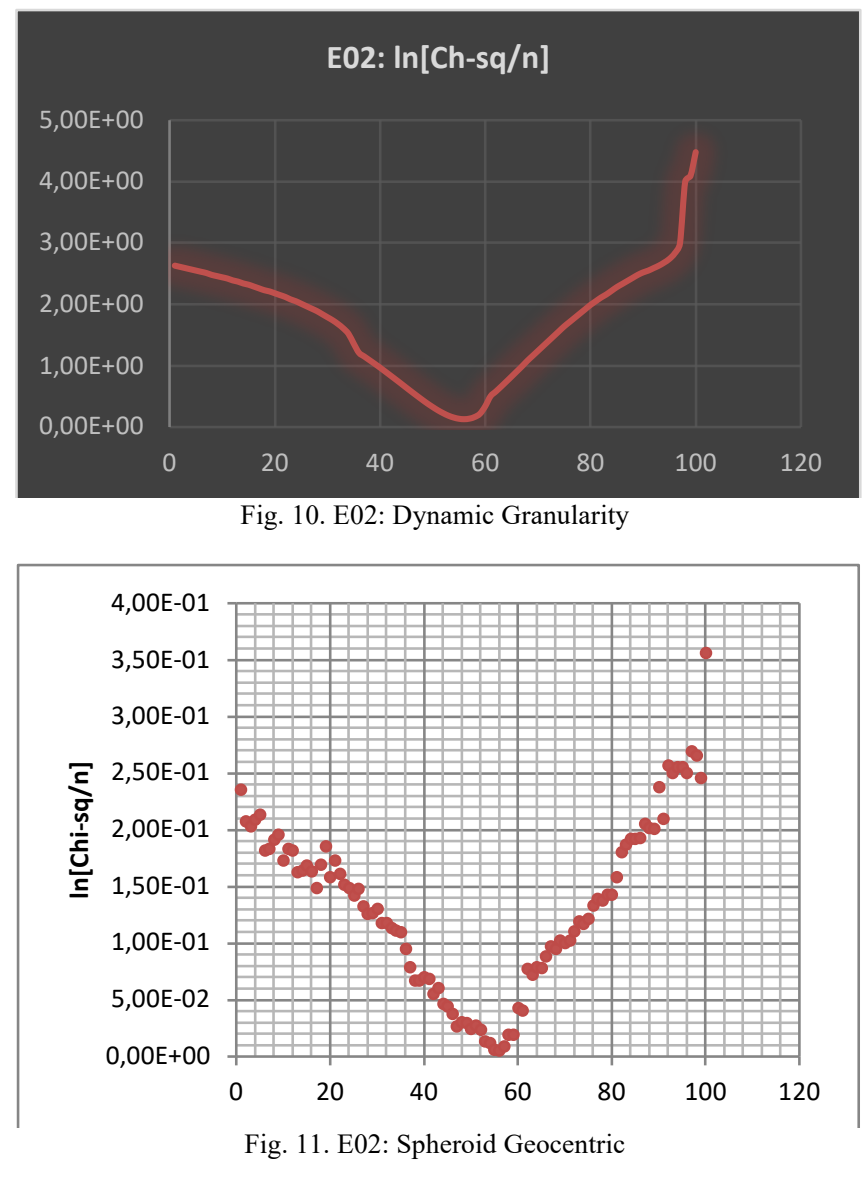

$6,00 E+00$

$5,00 E+00$

$4,00 E+00$

$3,00 E+00$

2,00E+00

$1,00 \mathrm{E}+00$

$0,00 E+00$

$-1,00 \mathrm{E}+00$

$-2,00 E+00$
Fig. 10. E02: Dynamic Granularity

Fig. 11. E02: Spheroid Geocentric

\section{E05: In[Chi-sq/n]}




\section{E05: $\ln [$ Chi-sq/n]}

$6,00 E+00$

$5,00 E+00$

$4,00 E+00$

$3,00 \mathrm{E}+00$

$2,00 \mathrm{E}+00$

$1,00 E+00$

$0,00 \mathrm{E}+00$

$-1,00 E+00$

$-2,00 \mathrm{E}+00$

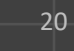

40

60
80

100

120

Fig. 13. E05 Dynamic Granularity

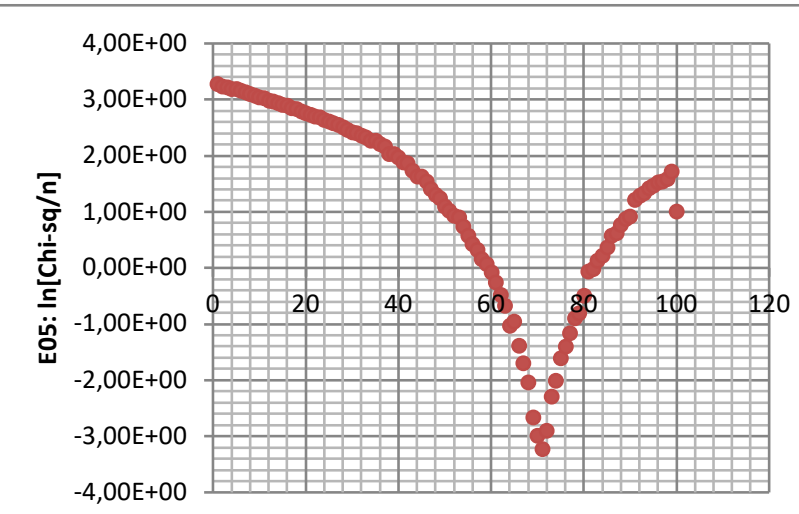

Fig. 14. E05 Spheroid Geocentric

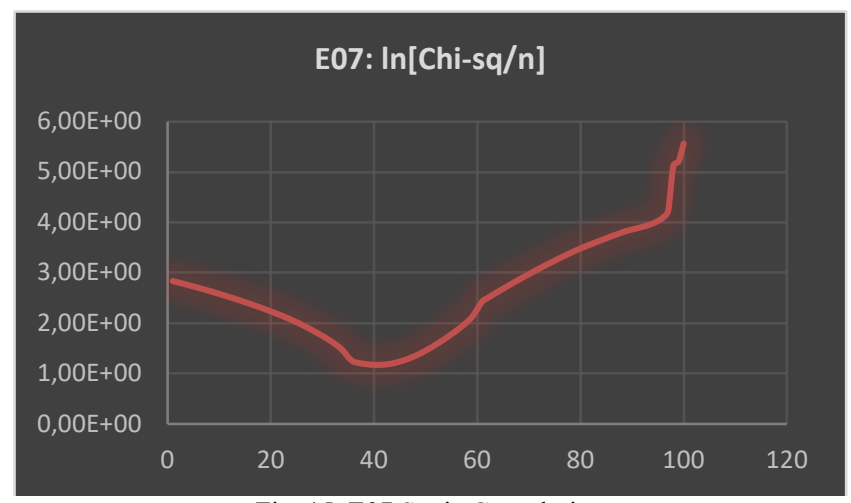

Fig. 15. E07 Static Granularity

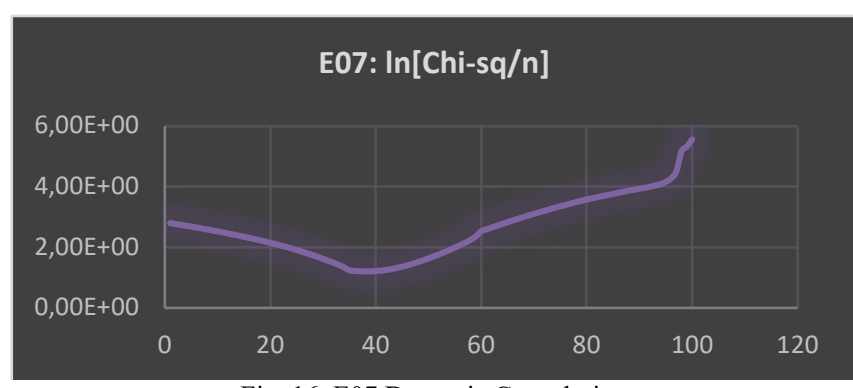

Fig. 16. E07 Dynamic Granularity

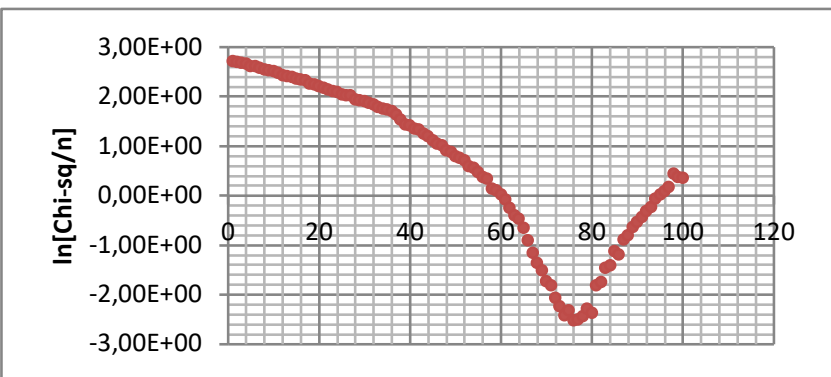

Fig. 17. E07 Spheroid Geocentric

\section{Discussion of Results}

It was noted that the conformal method, in the case of E02, processed, using the dynamic technique, one depth-point's station-set in approximately a mean time of 1.2 seconds; and using the static technique: 4.8 seconds; whereas the spheroidal model processed this in an approximate period of $123.6 \mp 3.65$ seconds. The cardinal number for the stationset in this case was 32 . These periods vary according to depth, epicenter-station distance and to processor used. However, the conformal method used only a spherical model of the Earth, while the latter incorporates the Earth spheroidal geometry. Therefore, we can expect the results of the latter to approach reality more closely, while the former will give a good sense of the result, generally within order of the spheroidal method.

It is found that the two forms follow a similar trend: for the Spheroidal, E02 is deeper than E05 and E07 is shallower than both, otherwise, in the Conformal mode, E02 is deeper than E05 but E07 appears deeper than both. In the following short analysis, the figures are still tentative given the current maturity of the programming.

\begin{tabular}{cccl}
\multicolumn{4}{c}{ TABLE IV: MEAN AND STANDARD DEVIATION FOR INDICATOR SET } \\
\hline \hline Static Granularity & Dynamic Granularity & Spheroidal & Earthquake \\
\hline $502 \mp 55.7 \sigma$ & $541 \mp 41.7 \sigma$ & $454 \mp 81 \sigma$ & E02 \\
\hline $440 \mp 88.4 \sigma$ & $457 \mp 93.3 \sigma$ & $445 \mp 135 \sigma$ & E05 \\
\hline $676 \mp 192 \sigma$ & $681 \mp 183 \sigma$ & $317 \mp 167 \sigma$ & E07 \\
\hline \hline
\end{tabular}

The values in Table IV are the Hypocenter depths estimated by each method for the three Earthquakes. The figures causing the observed large spreads can be seen from the variation of the estimates arising from the individual indicators [resumed at Table II]. Although each estimator, intuitively, should provide a minimum [or maximum] in its response to the whole scan, work is yet to be done on the sensitivity of individual indicators [see Table III] to error.

TABLE V: COMPARISON OF THE OUTPUT OF EACH METHOD [A =

\begin{tabular}{|c|c|c|c|}
\multicolumn{5}{|c|}{ STATIC; B=DYNAMIC; C SPHEROIDAL $]$} \\
\hline A to B & A to C & B to C & Earthquake \\
\hline $17.5 / 0.05$ & $54 / 0.01$ & $97 / 0.01$ & E02 \\
\hline $3.3 / 0.9$ & $66.3 / 0.01$ & $72.9 / 0.01$ & E05 \\
\hline $3.9 / 0.9$ & $1163 /<0.01$ & $1247 /<0.01$ & E07 \\
\hline
\end{tabular}

In Table V, the results of a Chi-squared test, performed between each indicator response profile for each method and for each Earthquake show appropriate similarities and differences. The figure before the slash is the chi-squared value, while the figure following, is the probability that the Null Hypothesis is true, viz: effectively, that the given response profile pair is similar.

The contents of this table show tight agreement between A and B for E05 and E07 and some agreement between A and $\mathrm{B}$ for E02. This may indicate, in the latter case, that the quality of the object data for E02 was worse than that for E05 and E07. There was no agreement between A \& C and B \& C for Earthquakes E02 and E05 but for Earthquake E07 an anomaly appeared, which has yet to be explained. See Table II for the figures. The lack of close agreement between A \& C and B \& C for E02 and E05 is most likely to be caused by the different Earth geometry model assumed between A \& B versus that of C, i.e. Spherical as opposed to Spheroidal. 
TABLE VI: COMPARISON OF EACH METHOD BETWEEN THE THREE EARTHQUAKES

\begin{tabular}{|c|c|c|c|}
\hline Static & Dynamic & Spheroidal & Earthquake \\
\hline $140 / 0.01$ & $170 / 0.01$ & $30.76 / 0.01$ & E02 to E05 \\
\hline $462 / 0.01$ & $345 / 0.01$ & $466 / 0.01$ & E02 to E07 \\
\hline $854 / 0.01$ & $576 / 0.01$ & $314 / 0.01$ & E05 to E07 \\
\hline
\end{tabular}

Table VI again shows data from a set of Chi-squared tests using the same format as Table V. Each Earthquake pair is shown to be distinct up to at least the 0.01 level of significance, and most importantly the pair: E02 and E05.

\section{CONCLUSION}

For Earthquakes E02 and E05, it appears that the degree of agreement between the Conformal methods and the Spheroidal is moderated by the difference in Earth geometry assumptions (vid. sup.). However, there is, still, an unexplained "anomaly" concerning E07.

Further, knowledge of the more detailed behavior of the members of the Indicator set will become apparent after more research.

Despite the anomaly with Earthquake E07 it seems that further research into the Conformal scanning concepts may be profitable.

\section{ACKNOWLEDGEMENTS}

Acknowledgement is made to IRIS (Incorporated Research Institutes for Seismology) for providing the Earthquake data, present and used in this paper, from the Database handled by the system Wilber 3 .

\section{REFERENCES}

[1] Daglish G. R. \& Sizov Yu. P. "Localisation of Earthquakes and their Tracking in Real-time", Chapter 9. Lambert Academic Publishers. 2019.

[2] Daglish G. R. \& Sizov Yu. P. "Assessment of results Produced by an Algorithm Delivering Earthquake Epi- \& Hypocenters Concurrently and in Real-time". Earth Science Research; Vol 4, No 1: 2015

[3] Daglish G. R. "A very Rapid Scan for Hypocenter Foci Driven by Conformal Transformation". Conference on Artificial Intelligence and Advanced Manufacturing”. (Dublin), ACM. 2019

[4] Daglish G. R. \& Sizov Yu. P. "Localisation of Earthquakes and their Tracking in Real-time", Chapter 10. Lambert Academic Publishers. 2019.

[5] Daglish G. R. \& Sizov Yu. P. "Real-time Earthquake Localization and the Elliptic Correction". Advances in Intelligent Computing. Springer. 2018

[6] Daglish G. R. \& Sizov Yu. P. "Localization of Earthquakes and their Tracking in Real-time", Annex I. Lambert Academic Publishers. 2019.

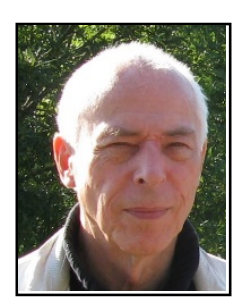

\section{George R. Daglish [06/03 1942]}

Having traveled extensively in N. Nigeria and Norway, he gained a BA (II.1 Hons, 1982) with the Open University (England) and subsequently a Doctorate in Applied Mathematics at Birkbeck College (London, 1992). He worked in Industry (Defense \& Oil Exploration) and Academia (Structures) and is now retired. With a past interest in tracking systems, he sees Earthquake Localization as providing a challenge, since it presents "noncooperating targets within highly non-linear reference and measurement spaces". His aim now is to construct Real-time Algorithms for Earthquake Localization and to work towards the "deconvolving" of multiple Earthquake events with interleaved energy onsets. Following this, he is attempting to produce fast real-time algorithms for Tsunami prediction 